\title{
Late onset Darier's disease: clinical and dermoscopic features
}

\begin{abstract}
Darier disease is an uncommon autosomal dominant genodermatosis with complete penetrance and variable expressivity. In the majority of cases, it develops from childhood and persists through adolescence. Clinically, it mainly manifest as hyperkeratotic papules predominating in seborrheic areas and flexures with associated nail abnormalities. The lesions are often associated with itching and malodor, which affects social living of these patients. Dermoscopy may be useful in improving its clinical recognition. Nevertheless, diagnostic confirmation is based on histology that shows characteristic features. There are currently no validated curative treatments, with the majority of cases treated symptomatically. However, oral retinoids may reduce discomfort by decreasing hyperkeratosis. We report a rare case of a late onset Darier's disease in a 61year old female with no familial history of such disease, with a special interest of its clinical and dermoscopic features. We also highlight the importance of dermoscopy as a helpful tool in recognizing Darier's disease lesions.
\end{abstract}

Volume 2 Issue 5 - 2018

\author{
Ghita Senhaji, Ouiame El Jouari, Salim \\ Gallouj, Amina Lamouaffaq, Fatima Zahra \\ Mernissi \\ Department of Dermatology, University hospital Hassan II, \\ Morocco
}

\section{Correspondence: Senhaji Ghita, Doctor, Department of Dermatology, University hospital Hassan II, Fez, Morocco, Tel +21260007 5857, Email ghitasenhaji88@gmail.com}

Received:September 12, 2018| Published: October 04, 2018

Keywords: darier disease, dyskeratosis follicularis, genodermatosis, dermoscopy, dyskeratosis, acantholysis

\section{Introduction}

Darier disease, also known as 'Darier-White disease' or 'dyskeratosis follicularis', is an uncommon autosomal dominant genodermatosis with complete penetrance and variable expressivity. ${ }^{1,2}$ It mainly manifest as hyperkeratotic, firm papules predominating in seborrheic areas and flexures with associated nail abnormalities. ${ }^{3}$ However, It is often under-diagnosed because of the paucity of lesions. ${ }^{2}$ Skin signs may be associated with itch and a distinct odor, which could lead to discomfort and social handicap. ${ }^{1}$ We present a rare case of Darier's disease with late onset in a 61year old female with no familial history of identical disease. In this observation, we detail clinical and dermoscopic features of this rare disorder.

\section{Case report}

A 61 year-old female patient, with no medical or familial history, presented to the Department of Dermatology of our Hospital, with a 3year history of persistent pruriginous and malodorant papules on the body. These lesions were insidious in onset and were neither painful nor associated with burning sensation. They became more itchy and infected during summer. There were no associated systemic complaints, nor any family history of hereditary diseases. Physical examination showed a grayish colored warty and hyperkeratotic papules and plaques, with rough and spiny surface and fetid odor, localized on post auricular areas, neck, intermammar folds, along with subungueal keratosis and trachyonychia (Figure 1) (Figure 2). There was no mucosal involvement. Dermoscopy showed yellowish polygonal structures of various size surrounded by a thin whitish halo (Figure 3). Systemic examination didn't reveal any abnormality. An incisional biopsy from the lesions was taken for microscopic examination which showed characteristic histopathological findings consistent with the diagnosis of Darier's disease (Figure 4). Given the presence of highly suggestive lesions with clinical and histopathological examination, a final diagnosis of Darier's disease was made. The patient was then treated with systemic alitretinoin with no major side effects. She was also encouraged to avoid triggering factors such as UV exposure, heat, and trauma and to daily apply topical emollients. She is on constant review and follow-up with mild improvement.

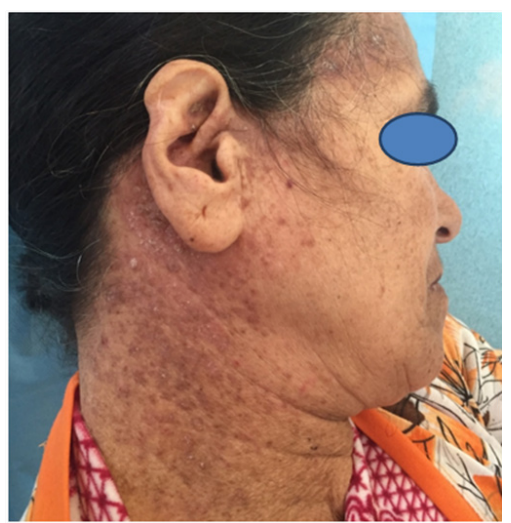

Figure I Multiple grayish warty and hyperkeratotic papules and plaques on post auricular areas and neck.

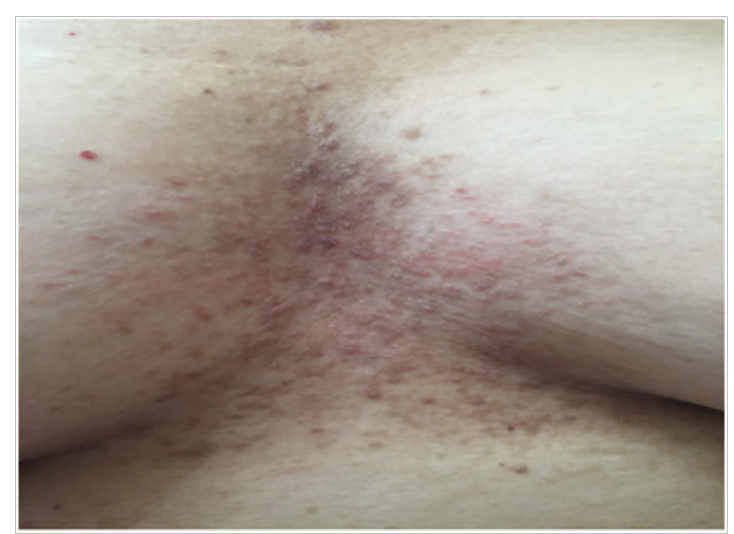

Figure 2 Multiple keratotic papules on the intermammar folds. 


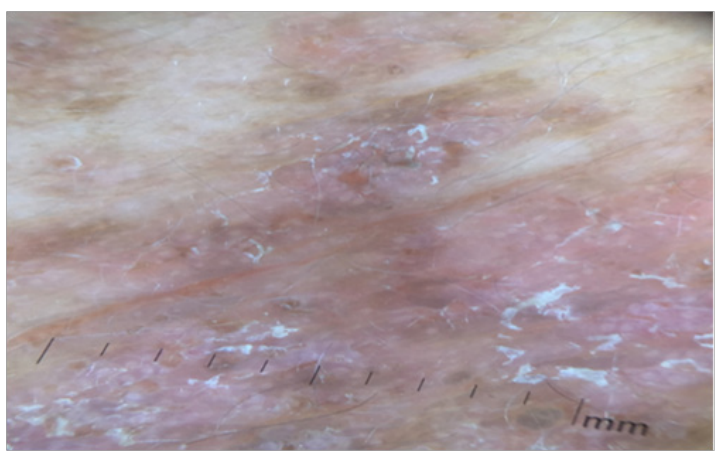

Figure 3 Dermoscopy of a hyperkeratotic plaque showing yellowish polygonal structures of various size surrounded by a thin whitish halo.

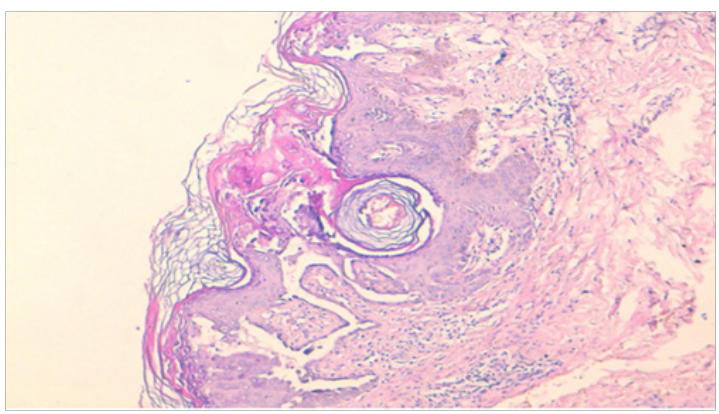

Figure 4 Histological picture showing hyperkeratosis, suprabasal clefts with acantholytic dyskeratosis, and corps ronds in the granular layer. (Hematoxylineosin, original magnification*40).

\section{Discussion}

Darier disease, also known as keratosis follicularis, is a rare autosomal dominant inheritance disease characterized by greasy hyperkeratotic papules in seborrheic regions, nail abnormalities and mucous membrane changes. ${ }^{4}$ In the majority of cases, Darier disease develops from childhood and persists through adolescence and has an equal gender distribution. ${ }^{5}$ Clinically, the distinctive lesion is characterized by hyperkeratotic papules that coalesce into plaques and occur primarily not only in seborrheic but also in intertriginous areas, as in our case. ${ }^{4}$ The lesions are often associated with itching and malodor. Additionally, complications such as maceration and secondary infection may result in a strong malodor. ${ }^{5}$ Associated abnormalities include nail abnormalities characterized by nail fragility, red and white longitudinal stripes and V-shaped notches at the free margin of the nails. ${ }^{4}$ However, no hair-related abnormalities are observed. ${ }^{5}$ Sun, heat and sweating exacerbate the disease. ${ }^{4}$ Dermoscopy may be useful in improving its clinical recognition and for distinguishing this disease from its other main clinical differential diagnoses. It mostly shows centrally located polygonal, star-like or roundish-oval shaped yellowish/brownish area, surrounded by a more or less thin whitish halo, overlying a pinkish homogeneous structure less area, with or without whitish scales and dotted and/or linear vessels presenting a whitish halo. ${ }^{6}$ Histology confirms the diagnosis showing characteristic features such as corp ronds, grains and suprabasal cleavage, ${ }^{5}$ as well as abnormal premature keratinization along with dyskeratosis and acantholysis in the horny layer. ${ }^{4}$

There are currently no validated curative treatments available for Darier disease, with the majority of cases treated symptomatically. ${ }^{5}$ However, oral retinoids may decrease hyperkeratosis, smoothen the papules and reduce odor, but should be used for a long period of time. ${ }^{4}$ Lifestyle advice is important in eliminating exacerbating factors, such as high temperatures, high humidity, UV rays and mechanical irritation. ${ }^{5}$ In fact, milder forms respond to general measures such as improvement of hygiene, wearing cotton clothes, avoidance of heat, sunlight and use of sunscreens. ${ }^{4}$ Additionally, antiseptic solutions such as triclosan or astringents are helpful. ${ }^{4}$ Other treatments include topical retinoids, calcipotriene, 5-fluorouracil, dermabrasion and photodynamic therapy. Moreover, ablative treatments such as $\mathrm{CO}_{2}$ and Erbium: YAG lasers have also been used in these conditions, with variable results and side effects. ${ }^{1}$ However, the lesions relapse because of the hereditary etiopathogenesis, especially in patients with the severe and generalized form of the disease. ${ }^{4}$

It is debatable whether the psychological effects this disease is due solely to the fact of unpleasant presence of "smelly-dirty "cutaneous lesions. DD gene has pleiotropic effects in skin and brain. Both tissues share an ectodermal origin and intracellular calcium signalling in neurons is involved in neuronal excitability and neurotransmission. ${ }^{7}$ There are numerous reports of cases of DD with neuropsychiatric disorders: epilepsy and depression. ${ }^{8}$

\section{Conclusion}

To sum up, we present a rare case of a late onset Darier disease. Through our observation, we discuss the clinical and histological aspects of this uncommon disorder. We also highlight the importance of dermoscopy as a non-invasive tool that may be useful in the diagnosis of this disease, and could help us ruling out other dermatosis with similar clinical aspect.

\section{Acknowledgements}

To the staff of the dermatological department of the Moroccan University Hospital Hassan II.

\section{Conflict of interest}

The authors declare no financial or any conflict of interest.

\section{Patient consent form}

Patient consent related case report take the permission from concerned patient.

\section{References}

1. Cannarozzo G, Bonciani D, Sannino M, et al. Dye Laser Treatment for Darier Disease: Results of a Case Series. Photomed Laser Surg. 2016;34(7):305-307.

2. Linder D, Marinello E, Donisi PM, et al. Inframammary Dermatitis: A Case of Localized Late-Onset Darier's Disease. Case Rep Dermatol. 2016;8(2):189-192.

3. Dharman S, Arvind M. Darier's disease--oral, general and histopathological features in a 7 year old child. J Indian Soc Pedod Prev Dent. 2016;34(2):177-179.

4. Suryawanshi H, Dhobley A, Sharma A, et al. Darier disease: A rare genodermatosis. J Oral Maxillofac Pathol. 2017;21(2):321.

5. Takagi A, Kamijo M, Ikeda S. Darier disease. $J$ Dermatol. 2016;43(3):275-279.

6. Errichetti E, Stinco G, Lacarrubba F, et al. Dermoscopy of Darier's disease. J Eur Acad Dermatol Venereol. 2016;30(8):1392-1394.

7. Baba-Aissa F, Raeymaekers L, Wuytack F, et al. Distribution and isoform diversity of the organellar Ca2+ pumps in the brain," Molecular and Chemical Neuropathology. 1998;33(3)199-208.

8. Munro CS. The phenotype of Darier's disease: penetrance and expressivity in adults and children. Br J Dermatol. 1992;127(2):126-130. 